\title{
Efectos de los árboles exóticos y del ambiente materno sobre la producción de semillas, la germinación y el crecimiento inicial de Polylepis subtusalbida (Rosaceae) en el Parque Nacional Tunari, Bolivia
}

\author{
Edgar E. Gareca ${ }^{\bowtie}$; Yvonne Y. Martinez; Claudia Solis \& Luis F. Aguirre \\ Centro de Biodiversidad y Genética, Facultad de Ciencias y Tecnología, Universidad Mayor de San Simón, Cochabamba, \\ Bolivia.
}

\begin{abstract}
Resumen. Los bosques de Polylepis subtusalbida en la ladera sur del Parque Nacional Tunari (Cochabamba, Bolivia) son relictos de considerable superficie de vegetación nativa ubicados a 3200-3900 m. Estos bosques prestan servicios ecosistémicos clave, en especial a la ciudad de Cochabamba. Sin embargo, están muy fragmentados y coexisten con árboles exóticos como Pinus radiata y Eucalyptus globulus. El presente trabajo evalúa el efecto de los árboles exóticos y del ambiente materno sobre la producción de semillas, y, en invernadero, la germinación y crecimiento inicial de Polylepis. Se estudiaron tres escenarios para el efecto de árboles exóticos: bosques de Polylepis a) rodeados por pajonal semi-natural, b) rodeados por árboles exóticos y c) mezclados con árboles exóticos. Para evaluar el porcentaje de frutos con semillas completas por árbol (una estimación de la producción de semillas) se utilizaron frutos de 67 árboles madre de siete fragmentos. La germinación y el crecimiento inicial se evaluaron con un diseño completamente aleatorio (germinación) y de bloques completos al azar (crecimiento). Las variables del ambiente materno se redujeron mediante PCA y se evaluó el efecto de los árboles exóticos, de las características seleccionadas de los árboles madre y de su micrositio sobre la producción de semillas, la germinación y el crecimiento en invernadero, con una selección de modelos desde atrás y modelos lineares mixtos. El porcentaje de frutos con semillas completas por árbol disminuyó a medida que aumentó la proporción de suelo desnudo bajo el árbol madre. La germinación fue alta y no resultó afectada por los factores estudiados. El crecimiento en altura de la progenie fue afectado por los árboles exóticos, por el porcentaje de suelo desnudo, por la cobertura de dosel y por la sanidad de los árboles madre. Se identificó a la producción de semillas como fase limitante de la regeneración de Polylepis. Por su parte, en invernadero, los árboles exóticos y las condiciones del ambiente materno afectaron al crecimiento inicial de su progenie (efecto materno).
\end{abstract}

[Palabras clave: aprovisionamiento de semillas, crecimiento temprano, disponibilidad de recursos, efectos maternos, efectos ambientales maternos, micrositio, regeneración]

\begin{abstract}
Aвstract. Effects of exotic trees and maternal environment on seed production, germination and initial growth of Polylepis subtusalbida (Rosaceae) at the Parque Nacional Tunari, Bolivia. Polylepis subtusalbida's woodlands at the Southern slope of Parque Nacional Tunari (Cochabamba, Bolivia) are key native vegetation remnants between 3200-3900 m.; they provide ecosystem services, specially to the city of Cochabamba. However, these woodlands are highly fragmented and coexist with exotic trees (Pinus radiata and Eucalyptus globulus). This work shows the effects of the exotic trees and the maternal environment on seed production, germination and initial growth of Polylepis. Three settings for the exotic trees were studied: Polylepis woodlands a) surrounded by semi-natural grassland, b) surrounded by exotic trees and c) mixed with exotic trees. Fruits from 67 mother trees belonging to seven fragments were used. The percentage of fruits with full seeds was measured as an estimation of seed production. Germination and early growth were evaluated in a common garden with a complete randomized design (germination) and a complete randomized block design (early growth). Variables from the maternal environment were reduced via PCA and backward multiple regression followed by general mixed linear models which were performed to evaluate the effect of the exotic trees, maternal traits and maternal microsite on the seed production, germination and early growth. The percentage of fruits with full seeds diminished as bare soil under the maternal tree increased. Germination was high and it was not affected by the studied factors. Early growth of the progeny was affected by the presence of the exotic trees, bare soil, leaf cover and health of the maternal tree. Seed production was identified as a limiting stage for Polylepis regeneration, and the exotic trees and maternal environment affected early growth of the progeny in a common garden (maternal effects).
\end{abstract}

[Keywords: early growth, maternal effects, environmental maternal effects, microsite, resource availability, seed provisioning, regeneration]

Editor asociado: Daniel Renison

$\triangle$ edgargareca.l@fcyt.umss.edu.bo 


\section{INTRODUCCIÓN}

La conservación de bosques nativos es un tema de relevancia mundial, fundamental para lograr el décimo quinto objetivo del milenio: "Proteger, restablecer y promover el uso sostenible de los ecosistemas terrestres, gestionar sosteniblemente los bosques, luchar contra la desertificación, detener e invertir la degradación de las tierras y detener la pérdida de biodiversidad" (Naciones Unidas 2015). Este tema se torna más importante en ecosistemas amenazados donde el objeto de conservación convive con especies arbóreas exóticas implantadas.

Las plantaciones de árboles exóticos se usan por su rendimiento, propiedades textiles y ornamentales (Pimentel et al. 2000). Sin embargo, y a pesar de que, en algunos casos, estas plantaciones pueden albergar y mantener flora y fauna nativa (Aguirre et al. 2007), también pueden afectar de forma negativa a las especies nativas (Everett 2000). En el caso de su efecto sobre árboles nativos, los principales procesos son de competencia por explotación de recursos o por interferencia pasiva, o cambios en las interacciones planta-animal como la depredación (Reigosa et al. 2004; Gareca et al. 2007a). Asimismo, en algunos escenarios, las especies exóticas pueden interferir el flujo de polen y de semillas de los árboles nativos, y así modificar características genéticas de las poblaciones de árboles nativos que son clave para desarrollar estrategias y medidas de restauración y conservación de estos bosques (Thomas et al. 2014).

El método más empleado para propagar árboles con fines de restauración de bosques es el establecimiento de plantines (Zahawi and Holl 2014), y la manera más común para su obtención es colectar semillas y hacerlas crecer por un tiempo en invernaderos (2 meses a 1 año), con la ventaja de que se minimizan las pérdidas por fallas en la germinación, por depredación de semillas y por mortalidad de plantines jóvenes (Holl 2012). Además, en el invernadero se pueden realizar experimentos de jardín común (en uno o muchos lugares), en los que se recolectan semillas de árboles que crecen en diferentes ambientes y se hace crecer su progenie; en estas condiciones, toda la progenie está sujeta al mismo ambiente y las diferencias entre árboles se deben principalmente a diferencias genéticas (White et al. 2007). Estos experimentos sugieren que los efectos del ambiente materno, las características del microclima y el micrositio en que se encuentran las plantas madre determinan la germinación y el crecimiento inicial de su progenie (Roach and Wulff 1987). Es más, los efectos del ambiente materno sobre su progenie pueden estar sujetos a selección natural para que la experiencia materna se traduzca en variación fenotípica adaptativa de su progenie, siempre que los estímulos ambientales experimentados por la madre sean similares a las condiciones que experimentará la progenie (Mousseau and Fox 1998; Wolf and Wade 2009).

En la zona altoandina, desde Venezuela hasta la Argentina, los remanentes de bosques suelen estar dominados por árboles del género Polylepis Ruiz and Pav. A pesar de estar fragmentados, estos bosques albergan una biodiversidad elevada, incrementan las precipitaciones locales, mejoran la captación de agua, regulan la escorrentía, controlan la erosión, retienen sedimentos y nutrientes, y son fuente de aprovisionamiento de recursos para las comunidades de seres humanos que viven en sus alrededores (Fjeldså and Kessler 1996; Gareca et al. 2010). Precisamente, por el uso antrópico de estos bosques, la expansión de la frontera agrícola y el cambio de uso de suelo respectivo es que los bosques de Polylepis son uno de los ecosistemas montanos más amenazados de América del Sur (Kessler 1995; Navarro et al. 2005).

Estudios previos sobre el efecto del ambiente materno de Polylepis australis Bitter determinaron que el paisaje no está relacionado con la viabilidad de las semillas, mientras que los suelos sin disturbios, con menos erosión y más cantidad de pajonal presentaron una viabilidad mayor de sus semillas (Renison et al. 2004). Además, las variables del mesositio de la planta madre de $P$. australis y el grado de conservación del bosque al que pertenecía ésta afectaron al crecimiento inicial de sus plántulas en campo (Renison et al. 2005).

Polylepis subtusalbida (Bitter) M. Kessler and Schmidt-Leb. (Kessler and Schmidt-Lebuhn 2006a), conocida localmente como kewiña, forma relictos boscosos en la ladera sur del Parque Nacional Tunari (PNT; Cochabamba, Bolivia) muy próximos a la ciudad de Cochabamba. Estos bosques brindan servicios ecosistémicos clave para la ciudad y están constituidos por uno de los pocos árboles nativos que habitan en la Cordillera por sobre los $3000 \mathrm{~m}$. Al igual que otras especies del género, $P$. subtusalbida está categorizada como vulnerable, y en Cochabamba, sus remanentes 
de bosque tienen la particularidad de coexistir con especies exóticas arbóreas como Eucalyptus globulus L'Her. (eucaliptos) y Pinus radiata L. (pinos). Estos árboles exóticos fueron plantados en los años 70s para proteger a la ciudad de Cochabamba de las inundaciones (Paz 1992), y actualmente forman diferentes escenarios con Polylepis. Los árboles exóticos rodean a los nativos o están mezclados con ellos, y generan condiciones que cambian la estructura y la composición de los bosques nativos (Agreda 2007; Gareca et al. 2007c). Sin embargo, en esta especie, y a nuestro conocimiento, no hay estudios relativos a los efectos del ambiente materno que podrían estar influyendo sobre su progenie $y$, por ende, sobre el futuro de Polylepis.

El objetivo del presente estudio fue determinar el efecto del ambiente materno, representado por la presencia de los árboles exóticos, las características de los árboles madre y su micrositio, sobre la producción de semillas, la germinación y el crecimiento inicial de plántulas de Polylepis subtusalbida en un jardín común. La hipótesis sostuvo que existe un efecto, tanto materno del micrositio como de las características del árbol madre, sobre las variables de respuesta, y que los árboles exóticos alteran este efecto.

\section{MATERIALES y MÉTODOS}

\section{Descripción de la especie}

Polylepis subtusalbida (Bitter) M. Kessler and Schmidt-Leb. (Kessler and Schmidt-Lebuhn 2006a) se distribuye en los alrededores de Cochabamba, en el centro de Bolivia; se trató anteriormente como $P$. incana Kunth subsp. subtusalbida Bitter en 1911 y P. besseri Hieron. subsp. subtusalbida (Bitter) M. Kessler en 1995. En una revisión realizada el año 2006, esta subespecie fue elevada a especie (Polylepis subtusalbida Kessler and Schmidt-Lebuhn 2006b). La especie comprende árboles con una altura de hasta $8 \mathrm{~m}$. Las hojas, que son imparipinnadas, poseen entre 1 y 3 pares de foliolos. Las flores son perfectas, de $\sim 1 \mathrm{~cm}$ de diámetro, protóginas y dispuestas en una inflorescencia tipo racimo (Simpson 1979). En la Cordillera del Tunari, alineada con la ciudad de Cochabamba, los bosques puneños de P. subtusalbida y Berberis commutata son la vegetación potencial desde los $3200 \mathrm{~m}$ hasta los $4000 \mathrm{~m}$. En esta zona de clima pluviestacional subhúmedo, el bosque potencial de $P$. subtusalbida actualmente está representado por fragmentos de diversa extensión y grado de estructuración, en muchos casos con dosel semiabierto o abierto, con pajonales sustituyentes. Esto los convierte en una prioridad para la conservación (Navarro et al. 2010).

\section{Sitios de muestreo}

Los sitios de muestreo se ubicaron en la ladera sur del PNT (17²0' S - 66 ${ }^{\circ} 10^{\prime} \mathrm{O}$ ) (Figura 1). Este parque nacional se creó en 1962 para proteger a la vegetación nativa, prevenir la erosión y evitar la formación de barrancos al norte de la ciudad de Cochabamba, así como para preservarlo como un espacio de recreación para su población (Rico 1996; Anónimo 2001). El rango de temperatura media en los sitios de muestreo varía entre $3.3^{\circ} \mathrm{C}$ y $23.9^{\circ} \mathrm{C}$ y la precipitación anual es $\sim 600$ mm (SERNAP 2000). Entre los 3200 y 4000 m, la vegetación de laderas montañosas, además de P. subtusalbida, presenta otros árboles como Citharexylum dentatum, Vallea stipularis, Morella pubescens, Escallonia schreiteri y E. myrtilloides. En el estrato arbustivo son frecuentes Berberis commutata, Schinus microphyllus, Berberis rariflora y Gynoxys psilophylla, entre otras, mientras que las especies herbáceas características del bosque son principalmente Bomarea crocea, Bomarea dulcis, Fuchsia apetala y Fuchsia boliviana (Navarro 2015). Sin embargo, debido a actividades humanas desarrolladas durante siglos (e.g., el uso indiscriminado del fuego, el sobrepastoreo y la preferencia por ganado vacuno y ovino), en la actualidad, el área de estudio está dominada por pajonales y arbustos de las etapas serales (Fjeldså and Kessler 1996; Navarro 2015) y por árboles exóticos implantados.

Las plantaciones con árboles exóticos, Pinus radiata y Eucalyptus globulus, se iniciaron a principios de la década del setenta para proteger a la ciudad de Cochabamba de las inundaciones (Paz 1992). Como resultado de las mismas, hoy, la ladera sur del PNT es un mosaico compuesto por pajonales serales, campos de cultivo y fragmentos de bosque de diferentes tipos: remanentes (dominados por Polylepis subtusalbida), fragmentos puros de Pinus radiata y fragmentos puros de Eucalyptus globulus. Esto genera fragmentos de $P$. subtusalbida rodeados por Pinus radiata o Eucalyptus globulus y fragmentos mixtos con una de las especies exóticas (Gareca et al. 2007c).

Para determinar el efecto de las especies arbóreas exóticas sobre las nativas se 


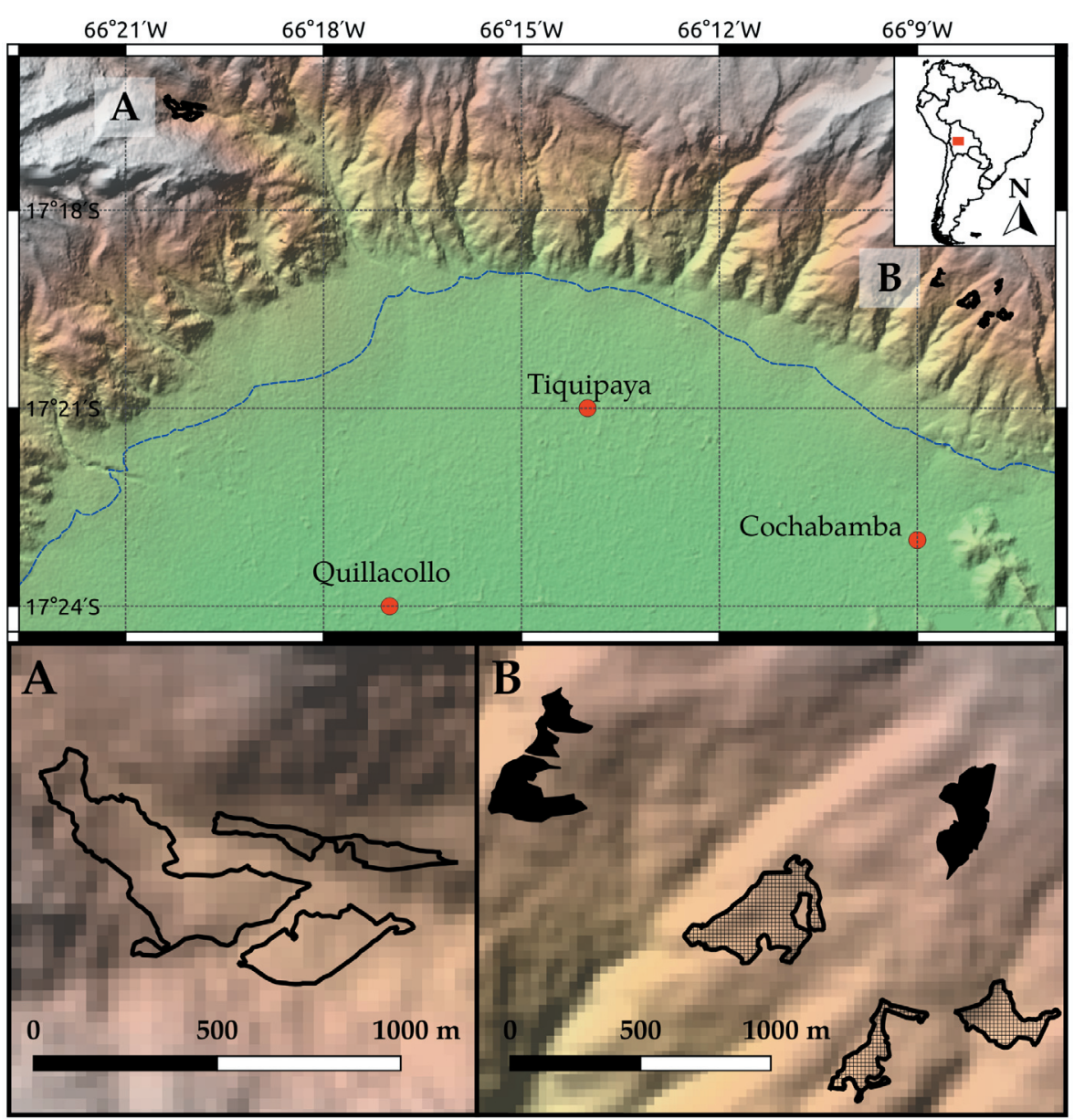

Figura 1. Mapa del área de estudio. Superior: ladera sur del Parque Nacional Tunari, su ubicación en América del Sur (recuadro), sitios con los escenarios estudiados (A y B), el límite sur del PNT (línea segmentada azul) y las principales poblaciones cercanas con sus nombres (círculos de color café). Inferior: (A) sitio de estudio A, con fragmentos de Polylepis rodeados por pajonal semi-natural (borde negro); (B) sitio de estudio B, con fragmentos de bosque de Polylepis rodeados por árboles exóticos (fragmentos con relleno punteado) y fragmentos de bosque de Polylepis mixtos con árboles exóticos (fragmentos con relleno sólido negro).

Figure 1. Study area map. Upper panel: southern slope of the Tunari National Park, its location in South America (inset), sites with the studied settings (A and B), southern limit of the PNT (dashed blue line), and the main towns (brown circles). Lower panel: (A) study site A, with Polylepis woodlands surrounded by semi-natural grassland (black border); (B) study site B, with Polylepis woodlands surrounded by exotic trees (hatched fragments) and Polylepis fragments mixed with exotic trees (black fragments).

seleccionaron: a) dos fragmentos de bosque de Polylepis puros rodeados por pajonal seminatural (PRP), b) dos fragmentos de bosques de Polylepis puros rodeados por especies arbóreas exóticas (PRE), y c) tres fragmentos de bosque de Polylepis mezclado con especies arbóreas exóticas (PME).

El área de los fragmentos seleccionados por escenario, su exposición, altitud y pendiente, así como la densidad de individuos de Polylepis en los mismos se detalla en la Tabla 1. Los fragmentos estudiados presentaron altitudes y densidades similares de Polylepis (excepto el fragmento PRP4), mientras que difirieron en el área, la exposición y la pendiente. Los fragmentos PRP presentaron también otras especies arbóreas (nativas) como Cytharexylum punctatum, Escallonia resinosa, E. myrtilloides, Schinus microphyllus y Vallea stipularis. En los tres fragmentos PRP, la mayor abundancia relativa fue de Polylepis subtusalbida ( $>65 \%$ ) (Agreda 2012). En cambio, los fragmentos del escenario PRE estuvieron rodeados por Eucalyptus globulus (fragmento 1) y por Pinus radiata (fragmento 3). A su vez, los tres fragmentos PME presentaron Eucalyptus globulus con densidades de 385, 429 y 227 individuos/ha para los fragmentos 1,2 y 3 , respectivamente, así como densidades 
Tabla 1. Área de los fragmentos estudiados, su exposición predominante, altitud (m), pendiente (\%) y densidad de individuos de Polylepis subtusalbida. En escenario, las siglas representan dos fragmentos de bosques de Polylepis puros rodeados de pajonal semi-natural (PRP), dos fragmentos de bosques de Polylepis puros rodeados por especies arbóreas exóticas (PRE) y tres fragmentos de bosque de Polylepis mezclado con especies arbóreas exóticas (PME).

Table 1. Studied fragments area, predominant aspect, elevation (m), slope (\%) and Polylepis subtusalbida density. In Escenario (setting), the acronyms stand for two pure Polylepis fragments surrounded by semi-natural grasslands (PRP), two pure Polylepis fragments surrounded by exotic trees (PRE), and three Polylepis fragments mixed with exotic trees (PME).

\begin{tabular}{lcccccc}
\hline Escenario & Fragmento & Área (ha) & Exposición & Altitud & Pendiente (\%) & Individuos/ha* $^{*}$ \\
\hline PRP & 3 & 12.1 & E & 3745 & 76 & 452 \\
& 4 & 2.9 & N & 3610 & 71 & 804 \\
PRE & 1 & 10.8 & O & 3610 & 44 & 481 \\
& 3 & 4.3 & O & 3630 & 49 & 468 \\
PME & 1 & 5.1 & SO & 3620 & 49 & 335 \\
& 2 & 2.7 & SO & 3720 & 44 & 371 \\
& 3 & 5.4 & SO & 3760 & 51 & 313 \\
\hline
\end{tabular}

${ }^{*}$ En base a datos de Agreda (2012)

de Pinus radiata de 21, 79 y 4 individuos por hectárea para los mismos fragmentos (Agreda 2012).

\section{Recolección de material vegetal}

La recolección de material vegetal se realizó en marzo y abril de 2013. En cada uno de los 7 fragmentos de bosque se seleccionaron al azar 10 árboles madre que presentaban frutos maduros y que rodeaban a un punto central. Cada uno de ellos fue georreferenciado, se identificó el escenario en el que se encontraba, el número de fragmento por escenario (1-3) y se le asignó un código de identificación. De cada árbol madre se recolectaron frutos maduros para realizar los experimentos en invernadero y se midieron las siguientes variables de cada árbol: diámetro del tronco tomado a $10 \mathrm{~cm}$ del suelo (o de cada tronco, en caso de haber más), área basal (que, en el caso de existir más de un tronco, se estimó como la sumatoria de las áreas basales de cada tronco del árbol correspondiente), altura, cobertura de dosel (evaluada con un densitómetro esférico forestal, Forestry Suppliers), área de la copa (estimada con la fórmula de una elipse y con los radios mayor y menor de la proyección de la copa sobre el suelo) y sanidad del árbol, evaluada en una escala de 1 a 3 (1: árbol con pocas hojas, muchas de ellas cloróticas o necróticas, débil, con hongos o presencia de insectos que pueden dañar su salud; 2: árbol intermedio entre 1 y 3; 3: árbol coposo, vigoroso, con hojas verdes y sanas, sin ramas rotas). En una parcela circular alrededor del tronco de cada árbol (área aproximada=3.5 $\mathrm{m}^{2}$ ) se estimaron visualmente los porcentajes de paja, arbustos, hierbas, roca, grava, suelo desnudo, hojarasca y musgo, así como la regeneración, medida como el número de plántulas entre 1 y $100 \mathrm{~cm}$ de altura.

\section{Germinación de Polylepis}

El experimento de jardín común se realizó desde julio a septiembre de 2013 en una de las platabandas del vivero del Centro de Semillas Forestales (BASFOR-ESFOR), con coordenadas $17^{\circ} 21^{\prime} 02^{\prime \prime} \mathrm{S}-66^{\circ} 09^{\prime} 16^{\prime \prime} \mathrm{O}$, y a una altitud de $2800 \mathrm{~m}$. La temperatura promedio durante los ensayos de germinación fue $16.6^{\circ} \mathrm{C}$, la mínima media fue $5.6^{\circ} \mathrm{C}$, la mínima absoluta fue $2.8^{\circ} \mathrm{C}$, la máxima promedio fue $25.1^{\circ} \mathrm{C}$ y la máxima absoluta, $30.3^{\circ} \mathrm{C}$. Como el presente trabajo es una base para la multiplicación y la producción de plantines de Polylepis en un jardín común, durante estos experimentos se emplearon las técnicas y condiciones de un potencial productor (BASFOR) con el fin de facilitar la germinación y la posterior producción en masa de plantines, que incluyó también al riego. El sustrato para la siembra contuvo $60 \%$ de arenilla y $40 \%$ de tierra negra. El sustrato fue esterilizado con formol y cubierto con plástico negro y mallas semi-sombra durante 7 días. Una vez que las platabandas estaban listas para la siembra se prepararon 108 parcelas de $20 \times 16 \mathrm{~cm}$, claramente identificadas.

Antes de la siembra se secaron los frutos a temperatura ambiente y en bolsas de papel, y se mantuvo la identidad del escenario (PRP, PRE y PME), del número de fragmento por escenario y del árbol madre. Una vez secos los frutos, se seleccionaron para ser sembrados aquellos con buena sanidad y sin evidencia de depredación. Para que las semillas germinaran más rápido, 24 horas antes de la siembra se remojaron los frutos preseleccionados. Los frutos de cada árbol madre se sembraron en una parcela identificada con el código del árbol madre y se las regó por inundación. Luego, para facilitar la germinación, la platabanda se cubrió por 3 semanas con el plástico negro y las mallas semi-sombra. 
Pasado este tiempo se evaluó la germinación una vez por semana durante 3 meses, con un riego de dos veces cada día. Una vez concluido el ensayo de germinación se recolectaron los frutos remanentes para estimar la producción de semillas. Se consideró como una plántula germinada a aquella que presentaba la emergencia de una radícula o cotiledón por sobre el sustrato, y se la marcó para evitar contar a la misma plántula dos veces durante el resto de la evaluación.

\section{Producción de semillas}

La producción de semillas de Polylepis se midió como la cantidad de semillas con endospermo (completas), divididas por el número total de semillas utilizadas en el ensayo de germinación y multiplicadas por 100. Es decir, se calculó como porcentaje de frutos con semillas completas por árbol. El valor del numerador se obtuvo después de cortar transversalmente los frutos remanentes del experimento de germinación y adicionar las plántulas germinadas, suponiendo que las semillas sin endospermo (vacías) no pueden generar una plántula. El número total de semillas utilizadas fue el número de frutos sembrados en el ensayo de germinación dado que cada fruto contiene sólo una semilla.

\section{Crecimiento inicial de Polylepis}

Cuando las plantas tuvieron 4-5 hojas se las trasplantó a la platabanda de crecimiento para su desarrollo. El diseño experimental fue de cinco bloques completos al azar, con 90 plantines por bloque (cada uno con su madre identificada). Cada plantín (progenie) estuvo en una bolsa individual de polietileno con $10 \mathrm{~cm}$ de diámetro y $18 \mathrm{~cm}$ de altura, con sustrato compuesto por 30\% de tierra negra, $30 \%$ de tierra vegetal y $40 \%$ de lama, que es el sustrato de crecimiento utilizado por BASFOR. El riego se realizó una vez al día. El crecimiento inicial se evaluó como la altura de cada planta después de 5 semanas de crecimiento.

\section{Análisis de datos}

La selección de modelos lineares se realizó con GLMSELECT. Los análisis de varianza se realizaron con el procedimiento MIXED. Ambos fueron implementados en SAS/STAT ${ }^{\circledR}$ v. 13.2 de SAS University Edition v. 3.6 (SAS Institute 2014).

Reducción de variables. El juego de datos de los árboles madre se dividió en dos grupos: a) variables de los árboles madre, que incluyó: área basal, área de la copa, altura del árbol, cobertura de dosel, sanidad (para este análisis como variable continua) y número de troncos a $10 \mathrm{~cm}$ del suelo, y b) variables del micrositio donde se encontraban los árboles madre: coberturas de arbustos, grava, herbáceas, pajonal, rocas y suelo desnudo.

Se redujo el número de variables, tanto de los árboles madre como del micrositio, por medio de análisis de componentes principales para evitar el problema de colinearidad en los análisis posteriores ( $\mathrm{R}$ versión 3.4.1., $\mathrm{R}$ Core Team 2017). A partir de esta información se seleccionaron gráficamente variables ortogonales que tuviesen los menores valores de correlación, para servir como variables explicativas en los análisis de producción de semillas, germinación y tasas de crecimiento.

Producción de semillas y germinación en jardín común. Ambas variables de respuesta, el porcentaje de frutos con semillas completas por árbol (estimación de la producción de semillas) y la germinación, se evaluaron con la misma metodología y de manera independiente en función de los efectos del escenario (PRP, PRE y PME), del factor aleatorio de los fragmentos anidados en el escenario que considera las diferencias entre fragmentos, las características seleccionadas del micrositio de cada árbol madre (porcentaje de pajonal, plantas herbáceas y suelo desnudo) y de los árboles madre (altura de la planta madre, cobertura de dosel y sanidad). Inicialmente, se introdujo el modelo con todos los factores principales y las interacciones entre el escenario y de la sanidad (ambas categóricas) con las variables continuas, así como interacciones triples seleccionadas. Como se partió del modelo más completo posible, se redujo este modelo mediante la eliminación de factores desde atrás y a pasos ('backward regression') utilizando el criterio de información de Akaike corregido para muestras pequeñas $(\mathrm{AIC} c$, por sus siglas en inglés) y garantizando que si una interacción era seleccionada deberían estar presentes en el modelo los factores principales de dicha interacción. El AICc resume la información en cada modelo considerando el tamaño de la muestra y el número de parámetros del mismo $\mathrm{y}$, además, corrige para los casos de muestras pequeñas. Valores más bajos de AICc indican mejores modelos (Quinn and Keough 2002). A pesar de las posibles correlaciones entre 
variables explicativas, se usaron las variables presentes en el mejor modelo seleccionado para la interpretación de resultados.

Una vez seleccionado el modelo estadístico se realizó un modelo linear mixto con el fragmento como factor aleatorio, se evaluó su cuadro de análisis de varianza con la suma de cuadrados tipo 3 y, en caso de encontrarse un efecto significativo de los escenarios o de la sanidad y no de su interacción con otra variable continua, se realizaron comparaciones planeadas o contrastes de un grado de libertad definidos a priori entre los tipos de escenarios, específicamente entre PRP vs. PRE y PRP vs. PME, y entre sanidad baja vs. media y media vs. alta. En cambio, si en el análisis de varianza existieron efectos significativos de una variable continua o de la interacción escenario ${ }^{*}$ variable continua o sanidad*variable continua, se obtuvieron los parámetros de las funciones y se las graficó en $\mathrm{R}$ utilizando el paquete ggplot2 (Wickham 2009). Antes del análisis se verificó la normalidad de la variable de respuesta y se realizaron las transformaciones necesarias. Una vez realizado el análisis se evaluó la normalidad de los residuales.

Crecimiento inicial en jardín común. Debido al diseño experimental de bloques completos al azar en el que se evaluó el crecimiento en altura de Polylepis, los modelos incorporaron los factores aleatorios de fragmento y bloque,

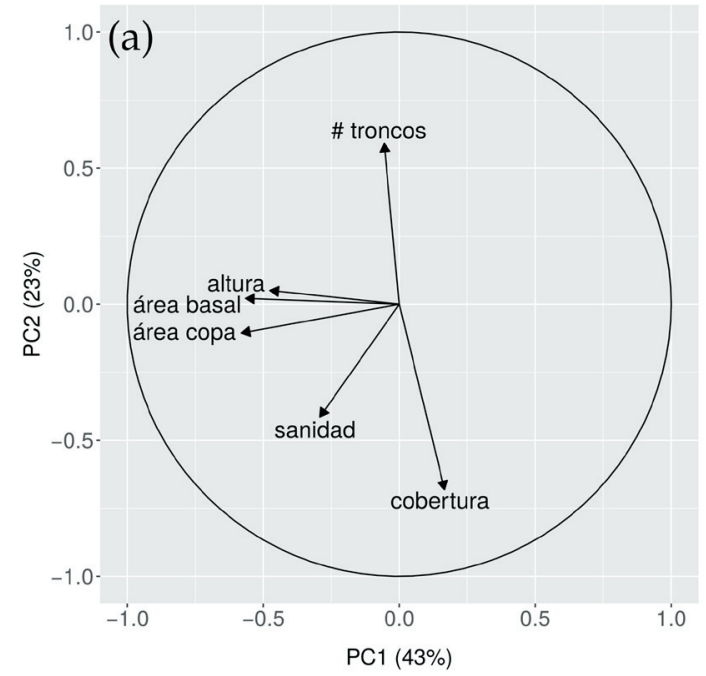

por lo que el análisis se realizó en el marco de los modelos lineares mixtos. Previo al análisis se evaluó el supuesto de normalidad de la tasa de crecimiento con diagramas de caja e histogramas. Posteriormente, con la mejor transformación se realizó la búsqueda del mejor modelo utilizando el AICc. Se comenzó con el modelo más completo, que incluía el efecto de bloques, de los escenarios, de las características seleccionadas para el micrositio y árboles madres, así como las interacciones entre los escenarios y las características de micrositio y árboles madre, además de algunas interacciones triples seleccionadas. Se fueron removiendo las variables con una selección desde atrás ('backward selection') hasta obtener el modelo reducido. Finalmente, en el caso de existir efectos significativos de un factor se procedió como en el caso de la germinación.

\section{RESULTADOS}

El análisis de PCA indicó que 81\% de la variación en las seis variables que presentan las características de los árboles madre (área basal, área de la copa, altura, cobertura de dosel, sanidad y número de troncos a $10 \mathrm{~cm}$ del suelo) se pueden resumir en los tres primeros componentes principales. Los PC1 y PC2, que representaron $56 \%$ de la variación, muestran que la altura, el área basal y el área de la copa de los árboles están muy relacionadas (Figura 2a).

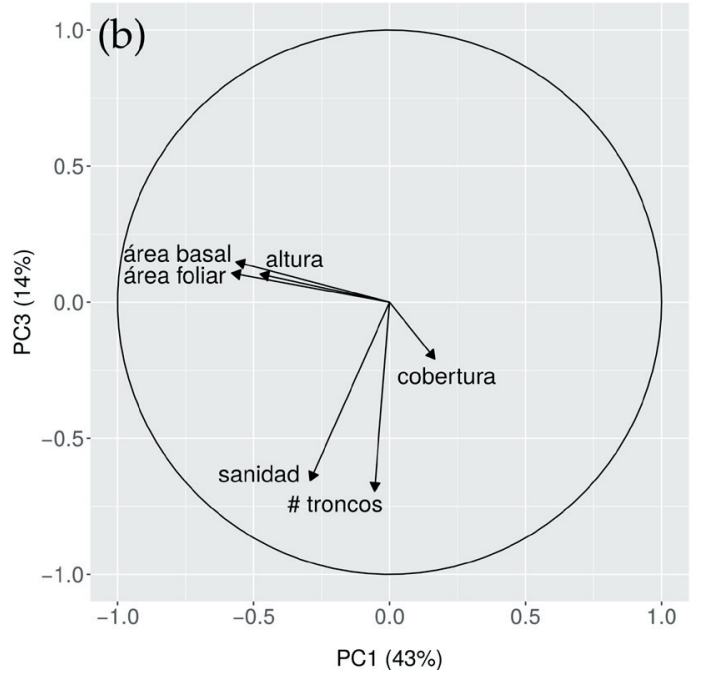

Figura 2. Características de los árboles madre dentro del círculo de correlaciones de los tres primeros componentes principales (PC) junto con la proporción de la varianza representada por cada componente. a) PC1 y PC2, b) PC1 y PC3. Las variables analizadas de los árboles madre fueron: área basal, área de la copa (área copa), altura, cobertura de dosel (cobertura), sanidad y número de troncos a $10 \mathrm{~cm}$ del suelo (\#troncos).

Figure 2. Kewiña mother tree characteristics in the correlation circles of the first three principal components with their explained variances (\%). a) PC1 and PC2, b) PC1 and PC3. The analyzed variables were: basal area (área basal), canopy area (área copa), height (altura), canopy cover (cobertura), health (sanidad), number of trunks at $10 \mathrm{~cm}$ above soil (\#troncos). 

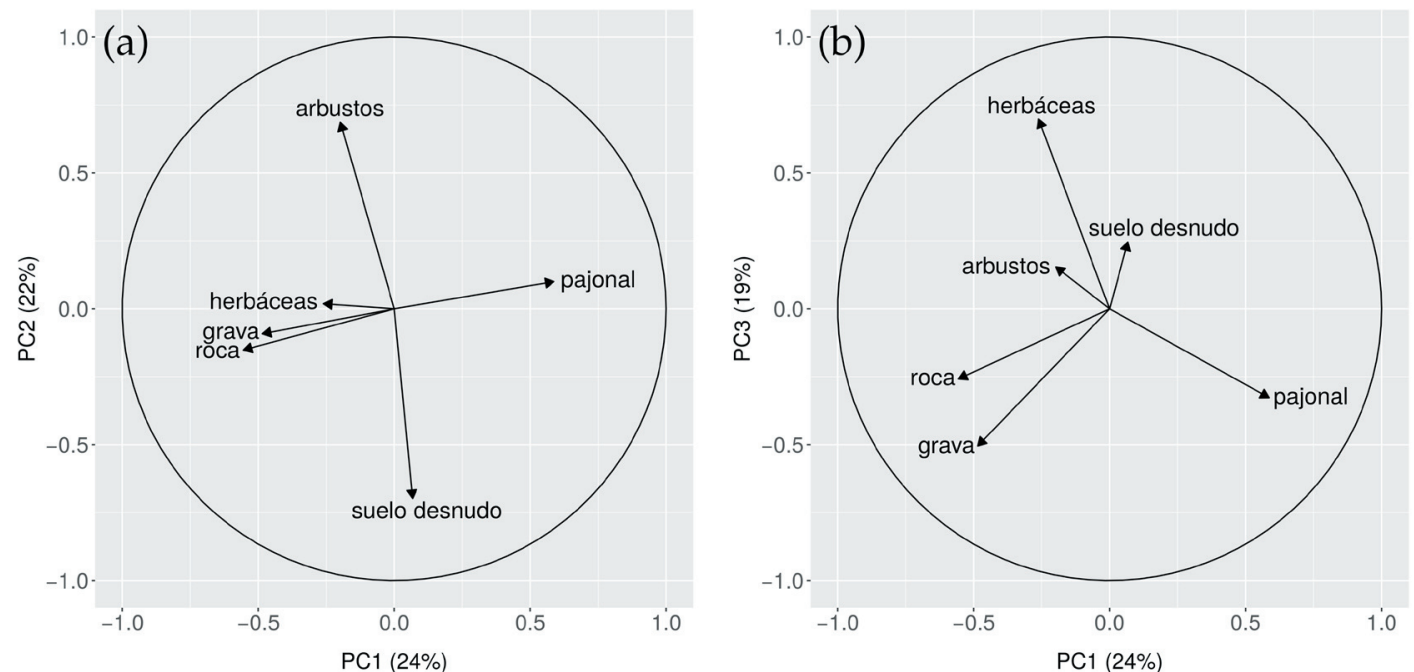

Figura 3. Variables del micrositio de los árboles madre dentro del círculo de correlaciones de los tres primeros componentes principales (a): PC1 y PC2, b): PC1 y PC3) junto con la proporción de la varianza representada por cada componente (\%). Las variables analizadas del micrositio de los árboles madre en porcentaje fueron las coberturas de: arbustos, grava, herbáceas, pajonal, rocas y suelo desnudo.

Figure 3. Microsite variables of Kewiña mother trees in correlation circle of the first three principal components (a): PC1 and PC2, b): PC1 and PC3) next to the variance explained by each component (\%). The analyzed microsite variables were the covers in percentages of: shubs (arbustos), gravel (grava), herbaceous plants (herbáceas), grasses (pajonal), rocks (roca), and bare soil (suelo desnudo).

Una variable independiente de las anteriores es la cobertura, mientras que la sanidad está asociada con el número de troncos (Figura 2b). Por consiguiente, se seleccionaron la altura del árbol, que es una medida de la habilidad competitiva de la planta (Weiher et al. 1999), la cobertura de dosel y la sanidad como variables representativas de las características de los árboles madre para análisis posteriores.

Respecto al micrositio de los árboles madre, el análisis de componentes principales de sus seis variables (porcentaje de arbustos, grava, herbáceas, pajonal, rocas y suelo desnudo) mostró que los tres primeros componentes acumularon $65 \%$ de la variación en estas variables. El análisis de los PC1 y PC2, que representó $46 \%$ de la variación, mostró que la cobertura del pajonal y del suelo desnudo son dos variables independientes, mientras que las coberturas de las herbáceas, grava y roca están relacionadas entre sí, y la cobertura de los arbustos está inversamente relacionada con la del suelo desnudo (Figura 3a). En cambio, al analizar la figura del PC1 y PC3 se observó que la cobertura de herbáceas fue la más asociada con el PC3 (Figura 3b). Por lo tanto, las variables que resumen el micrositio de los árboles madre fueron el suelo desnudo y la cobertura del pajonal y de las herbáceas. Estas variables se utilizaron para análisis posteriores.

Las correlaciones entre las características previamente seleccionadas del micrositio y de los árboles madre mostraron asociaciones bajas entre sí (Figura 4). El valor absoluto más alto de correlación fue el obtenido entre suelo desnudo y la altura de los árboles madre

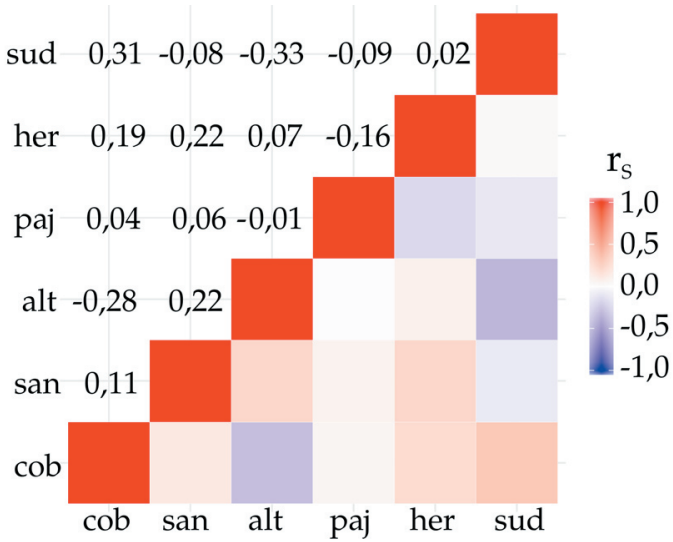

Figura 4. Valores de correlación de Spearman $\left(\mathrm{r}_{\mathrm{S}}\right)$ entre las variables correspondientes al micrositio de los árboles (coberturas de sud: suelo desnudo, her: plantas herbáceas y paj: pajonal) y las de los árboles (alt: altura, san: sanidad y cob: cobertura de dosel). Los colores rojo y azul indican valores positivos y negativos, respectivamente; la intensidad del color representa la magnitud de la correlación. Los valores de correlación se encuentran en la matriz triangular superior.

Figure 4. Heatplot of the Spearman correlations $\left(r_{c}\right)$ between the variables of the Kewiña tree microsites (sud: bare soil, her: herbaceous plants and paj: grasses) and the tree variables (alt: height, san: health and cob: canopy cover). Red and blue colors are positive and negative values, respectively. Intensity of the color represents the magnitude of the correlation. Correlation values are in the upper triangular matrix. 
$\left(r_{s}=-0.33, P<0.01\right)$, seguido de la relación entre suelo desnudo y cobertura del dosel $\left(\mathrm{r}_{\mathrm{S}}=0.31\right.$, $P=0.01)$.

El porcentaje máximo de frutos de Polylepis con semillas completas (con endospermo, medida de la producción de semillas) fue 53\%, mientras que el mínimo fue $0 \%$; la mediana, $14 \%$ y su promedio, 18\%. Cuatro árboles del escenario PME no presentaron semillas completas. El mejor modelo de regresión múltiple de todos los analizados que explicó al porcentaje de frutos con semillas tuvo como variable explicativa, además del escenario y del fragmento, al suelo desnudo (AICc $=144.53$, Material Suplementario Tabla MS1). De estas variables explicativas, el porcentaje de suelo desnudo fue la única que presentó un efecto significativo $\left(\mathrm{F}_{1,18.8}=4.16, P=0.05\right)$, mientras que las diferencias entre escenarios no fueron significativas $\left(\mathrm{F}_{2,3.93}=0.69, P=0.55\right)$. A mayor porcentaje de suelo desnudo se observó un menor porcentaje de frutos con semilla (Figura 5a), mientras que para los tipos de bosque, los errores estándar fueron muy grandes (entre 12 y 18 unidades porcentuales) (Figura 5b).

En cuanto a la germinación de semillas, el porcentaje medio de germinación fue $99.5 \%$. Cinco árboles presentaron una germinación menor a $100 \%$, y el valor mínimo de germinación observado en las semillas de un árbol del escenario PRP fue 91\%.

El mejor modelo para el crecimiento inicial de las plántulas de Polylepis en el jardín común incluyó, además de los factores aleatorios de

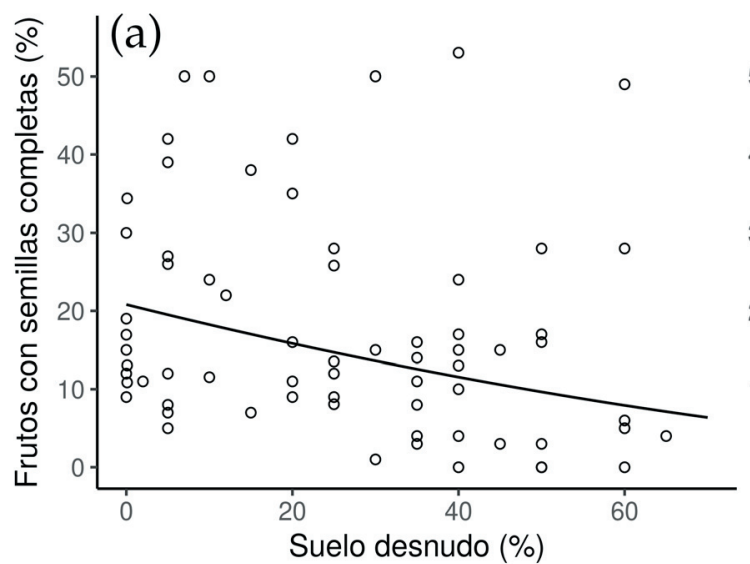

bloques y fragmentos anidados en el escenario, a los efectos fijos de escenarios, la cobertura de dosel, el suelo desnudo, la sanidad del árbol madre y las interacciones cobertura de dosel por escenario y suelo desnudo por sanidad (AICc=-119.07, Material Suplementario Tabla MS2). Los efectos aleatorios incluidos en el modelo fueron efectivos (estimación $>0$ ) y ambas interacciones fueron significativas (cobertura*escenario: $\mathrm{F}_{2,214}=6.31, P<0.01$, suelo desnudo*sanidad $\mathrm{F}_{2,214} \stackrel{2,214}{=}=7.55, P<0.01$ ) (Figura 6), por lo que sólo se interpretaron éstas y no sus efectos principales.

Primeramente, respecto a la interacción escenario por cobertura de dosel, el patrón de los escenarios menos afectados por las especies exóticas fue similar (PRP y PRE), con una pendiente igual a cero (PRP: $\mathrm{t}=-0.41, P=0.69 \mathrm{y}$ PRE: $t=-0.41, P=0.68)$. En cambio, el escenario más afectado por las especies exóticas (PME) presentó coberturas de dosel superiores a 60\% $\mathrm{y}$ un incremento positivo en el crecimiento inicial a medida que aumentó la cobertura de dosel ( $\mathrm{t}=2.46, P=0.01)$ para alcanzar un valor máximo similar al de los escenarios PRP y PRE. Finalmente, respecto a la interacción sanidad del árbol madre por suelo desnudo se observó que para los árboles madre con sanidad baja y media, el porcentaje de suelo desnudo afectó ligera, pero significativamente, a la altura de su descendencia (sanidad baja: $\mathrm{t}=-3.88, P<0.01$ y sanidad media: $\mathrm{t}=-2.16, P=0.03$ ). Mientras tanto, para los árboles madre con sanidad alta se observó que a mayor porcentaje de suelo desnudo hubo mayor crecimiento inicial en su descendencia $(\mathrm{t}=3.12, P<0.01)$.

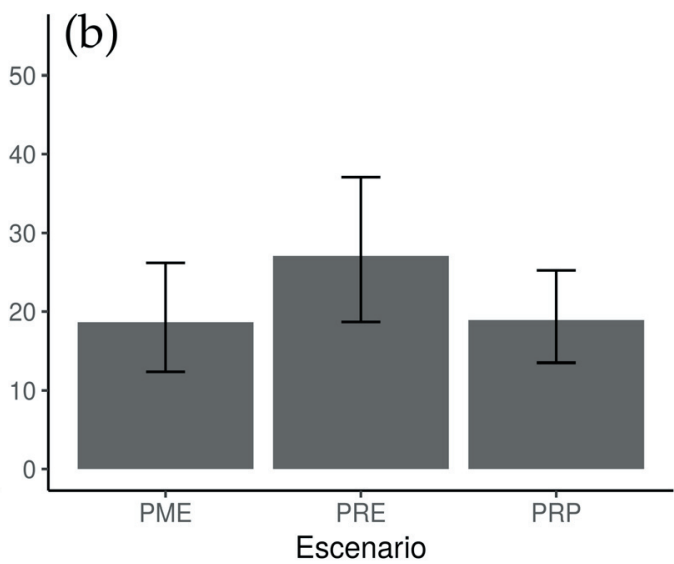

Figura 5. Producción de semillas, estimada a través del porcentaje de frutos de Polylepis con semillas completas por árbol, en función de a) el porcentaje de suelo desnudo bajo las plantas madre y b) el tipo de bosque en que se encuentran. La línea entera en (a) representa el mejor modelo linear ajustado para el suelo desnudo y las barras en (b), los errores estándar.

Figure 5. Seed production, estimated as percentage of fruits with full seeds, as a function of a) bare soil percentage under Polylepis mother trees and b) woodland type. The black line in (a) represents the best linear model adjusted for the effect of bare soil percentage, and the bars in (b) are standard errors. 

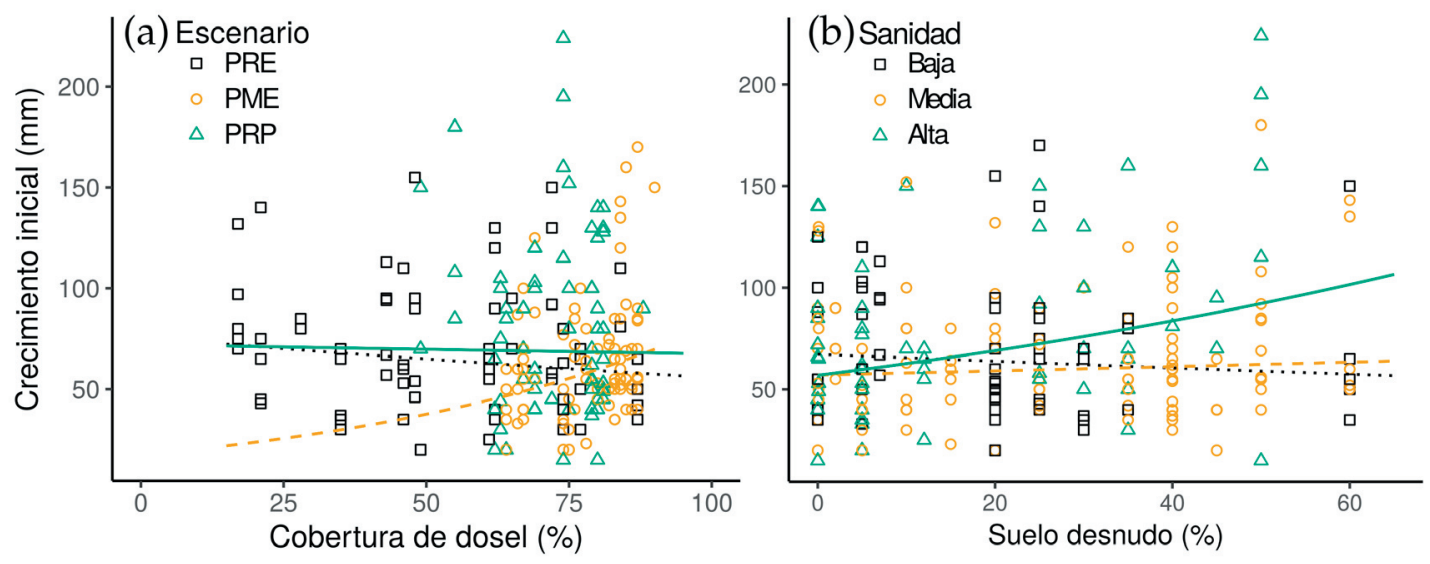

Figura 6. Crecimiento inicial de las plántulas de Polylepis en función de a) la cobertura del dosel y del escenario de la planta madre, y b) el porcentaje de suelo desnudo y la sanidad de la planta madre. PRE: Polylepis rodeado de árboles exóticos, PME: Polylepis mezclado con árboles exóticos y PRP: Polylepis rodeado de pajonal.

Figure 6. Polylepis early growth as a function of: a) leaf cover and setting, and b) bare soil and mother tree health. PRE: Polylepis fragments surrounded by exotic trees, PME: Polylepis fragments mixed with exotic trees and PRP: Polylepis fragments surrounded by grassland.

\section{DisCUSIÓN}

Los resultados obtenidos pueden resumirse como un bajo porcentaje de frutos con semillas completas de Polylepis asociado con el porcentaje de suelo desnudo bajo las plantas madre, y no con las características de los árboles madre ni con la presencia de árboles exóticos. La germinación fue muy alta y no resultó afectada por los árboles exóticos ni por las variables del árbol madre ni de su micrositio. En cambio, el crecimiento en altura de la progenie sí fue afectado por la presencia de los árboles exóticos, por el porcentaje de suelo desnudo bajo el árbol madre y por su cobertura de dosel y sanidad.

\section{Producción de semillas}

El 94\% de los árboles de Polylepis subtusalbida estudiados presentaron al menos un fruto con semilla completa (con endospermo), $\mathrm{y}$, en promedio, sólo $18 \%$ de los frutos de cada árbol mostró este tipo de semillas. Si, además, se considera que en este estudio no se utilizaron los frutos inmaduros, ni con presencia de hongos ni con signos de depredación, se puede comprender que este $18 \%$ sobre-estima el porcentaje de frutos con semillas completas reportado. Esto implica que si bien los Polylepis de la ladera sur del PNT aún pueden producir semillas, cada árbol, en promedio, produce pocas semillas completas. Aunque es raro que en las plantas haya una fecundidad de 100\% (es decir, que todos los óvulos generen semillas completas) este valor suele hallarse entre 30\% y $70 \%$ (Körner 2003). El bajo porcentaje de frutos con semillas completas por árbol puede ser una estrategia para incrementar la chance de dejar descendencia, posiblemente ante la fuerte depredación pre-dispersión que se observó en los frutos recolectados y que no se usaron en este estudio. En el caso del género Polylepis, los estudios de germinación reportan valores más bajos, y específicamente en aquellos casos en que se evaluó la presencia de semillas completas en los frutos, los valores medios oscilaron entre $10 \%$ y $23 \%$ para P. tomentella (Domic et al. 2017), $17 \%$ para P. besseri (Gareca et al. 2012) y entre $20 \%$ y $23 \%$ para $P$. australis (Renison et al. 2004; Seltman et al. 2007). Esto sugiere que el problema de Polylepis es el bajo porcentaje de frutos con semillas completas que tienen sus árboles.

El desarrollo de la semilla en la madre está frecuentemente limitado por recursos, por polen viable disponible, por incompatibilidad genética y por depredación pre-dispersión (Fenner and Thompson 2005). De estos factores, en el presente estudio se descarta la depredación pre-dispersión, debido a la preselección que se hizo de los frutos. A continuación, se analiza el efecto de los árboles exóticos, las características de las plantas madre y las de su micrositio sobre su porcentaje de frutos con semillas completas por árbol. Al estudiar qué variables afectaron al porcentaje de frutos con semillas completas por árbol de Polylepis, primeramente se encontró que las especies exóticas no la afectaron, pues hubo mucha variación en sus 
promedios. Por ejemplo, el error estándar del escenario PRE varío entre 19\% y 37\% (18\% de variación) y el error estándar máximo de PRP y PME fue $\sim 25 \%$, dentro del rango cubierto por los errores estándar del escenario PRE (Figura 5b). Este hecho llama la atención, pues se esperaba que las especies exóticas afectasen al porcentaje de frutos con semillas completas por árbol de Polylepis mediante competencia por explotación de recursos o interferencia pasiva (Reigosa et al. 2004), como la producción de sustancias alelopáticas (eucaliptos) o acidificación del suelo (pinos). Segundo, las características del árbol madre estudiadas no fueron seleccionadas como componentes del modelo para el porcentaje de frutos con semillas completas por árbol utilizando el criterio AICc (Material Suplementario Tabla SM1), por lo que no son predictores relevantes para la variación del porcentaje de frutos con semillas completas por árbol. Estas características podrían haber dado ventaja competitiva a los individuos, por ejemplo, a mayor altura, mayor disponibilidad de luz, mayor producción de fotosintatos y mayor porcentaje de frutos con semillas completas por árbol. Sin embargo, estas características no afectaron a esta variable. Finalmente, una de las características del micrositio, el porcentaje de suelo desnudo, sí afectó de forma negativa al porcentaje de frutos con semillas completas, por lo que en él estaría el o los factores limitantes para la producción de semillas de Polylepis en la ladera sur del PNT.

El suelo es la fuente de nutrientes para las plantas; la captura de nutrientes puede estar regulada por la disponibilidad de agua (que también nutre a la planta) y por las micorrizas. En este contexto, un suelo desnudo tiene mayor escorrentía y drenaje de agua que uno con cobertura vegetal (Lavelle and Spain 2001), por lo que tiene menor disponibilidad de agua para ser usada por las plantas. Además, la escorrentía y el drenaje del agua en suelos desnudos remueve nutrientes del suelo, lo que también disminuiría su disponibilidad. Por otro lado, las plantas herbáceas o arbustivas podrían competir con el árbol madre de Polylepis y disminuir su porcentaje de frutos con semillas completas por árbol. En cambio, la ausencia de especies herbáceas o arbustivas en sitios con altos porcentajes de suelo desnudo disminuiría la competencia por recursos, que estarían disponibles y Polylepis podría invertirlos para incrementar el porcentaje de frutos con semillas completas por árbol que se observó en el presente estudio. Acerca de las micorrizas, se sabe que sus comunidades pueden modificar la manera en que las plantas ubican sus nutrientes en las flores, frutos y semillas (Vivas et al. 2015). Consiguientemente, se encontró que en $P$. australis, la colonización por micorrizas arbusculares fue alta en comparación con otras especies de árboles, y que las mismas pudieron mejorar su desempeño (Menoyo et al. 2009). Asimismo, se encontró una asociación entre P. subtusalbida y micorrizas en Chorojo a $~ 60$ $\mathrm{km}$ de nuestro sitio de estudio (Hensen 1994). Por lo tanto, en suelos desnudos habría menos cantidad de nutrientes que en suelos con cobertura vegetal o menor competencia por los mismos, y su accesibilidad para Polylepis en la ladera sur del PNT estaría mediada por la disponibilidad de agua y, posiblemente, por micorrizas.

Estos resultados también están en línea con un estudio realizado para determinar las causas de la baja germinación de P. australis en el que se encontró una viabilidad mayor de las semillas a mayor cobertura de pajonal y menor erosión de suelo. Esto apoya la hipótesis de limitación de recursos (Renison et al. 2004). Por lo tanto, suponiendo que la polinización de Polylepis en el PNT es adecuada y que no hay incompatibilidad genética, los resultados presentados apoyan la hipótesis de limitación de recursos en Polylepis, lo cual estaría asociado con la disponibilidad de agua y de micorrizas.

\section{Germinación}

Considerando las semillas completas de $P$. subtusalbida colectadas del PNT, la germinación en el jardín común fue muy alta (99.5\%), con sólo 5 de 62 árboles que presentaron un porcentaje menor a $100 \%$ (dos de ellos en el escenario PRP, dos en el PRE y uno en el PME). Al contrario de lo que ocurrió con el porcentaje de frutos con semillas completas por árbol, que presentó una variación elevada entre escenarios, en este caso, la variabilidad fue muy baja como para realizar análisis estadísticos. A pesar de esta limitación, se puede indicar que los escenarios con especies exóticas, las características del micrositio y las de las plantas madre no modificaron la alta capacidad germinativa de $P$. subtusalbida en la ladera sur del PNT, por lo que no hay evidencia de un efecto materno sobre la germinación.

Al igual que en el caso del porcentaje de frutos con semillas completas por árbol, llama la atención que las especies exóticas 
no hayan afectado a la germinación de Polylepis en las condiciones homogéneas del jardín común, pues se esperaba que por explotación de recursos o interferencia pasiva afectasen a la progenie de los árboles nativos. De esta manera, se podría traducir en variación fenotípica adaptativa si los estímulos ambientales experimentados por la madre y la descendencia fuesen los mismos (Mousseau and Fox 1998; Wolf and Wade 2009; ejemplos en Roach and Wulff 1987). El hecho de no encontrarse evidencia que apoye esta hipótesis va en contra de estudios de germinación in vitro de P. subtusalbida realizados anteriormente en que se observó mayor germinación de semillas provenientes de bosques puros rodeados de pajonal (escenario PRP) que de las provenientes de bosques en mixtos (escenario PME, Gareca et al. 2007b, Martínez et al. 2006). A pesar de que puede haber variación interanual en los efectos maternos, es necesario saber bajo qué circunstancias estos se activan o liberan.

Por otro lado, al comparar los porcentajes de germinación obtenidos con los de otros estudios, es evidente que nuestra estimación de germinación es muy alta en relación con los valores reportados para otras especies de Polylepis, que varían entre $3 \%$ y $10 \%$ para $P$. subtusalbida (Hensen 1994; Gareca et al. 2007b), $10 \%$ para $P$. incarum (Vega et al. 2016), $10.4 \%$ para $P$. pacencis (Vega et al. 2016), entre $20 \%$ y $23 \%$ para $P$. australis (Renison et al. 2004; Seltman et al. 2007) ó $42 \%$ para P. neglecta (Vega et al. 2016). En el caso de la estimación previa de P. subtusalbida (Gareca et al. 2007b), se estimó la germinación considerando todos los frutos utilizados para los ensayos y sin descontar aquellos que tenían sus semillas vacías, como en el presente estudio. Para $P$. australis, aunque no se cuenta con los datos originales, se reportó que del $77 \%$ de semillas no viables, $94.5 \%$ estuvieron vacías (Renison et al. 2004) y que del $80 \%$ de semillas no viables, $98 \%$ estuvieron vacías (Seltman et al. 2007). De igual manera, se reportó que para P. incarum, $P$. pacensis y $P$. neglecta, los porcentajes de semillas vacías fueron $73 \%, 81 \%$ y $45 \%$, respectivamente. La mayor cantidad de semillas no viables estuvieron vacías en los diferentes estudios. El separar el porcentaje de frutos con semillas completas por árbol, estimación de la producción de semillas, de su germinación permite identificar qué proceso es más importante o limitante: la formación de las semillas (y los factores que la afectan) o su germinación (y los factores que la afectan). Esta separación es fundamental, pues los factores que afectan al porcentaje de frutos con semillas completas por árbol no son necesariamente los mismos que afectan su germinación.

\section{Crecimiento}

El crecimiento inicial de Polylepis en el PNT mostró evidencias de efecto materno mediado por la presencia de los árboles exóticos, del suelo desnudo, de la cobertura de dosel del árbol madre y de su sanidad. Estos resultados se esperaban, ya que el establecimiento (y el crecimiento) de las plántulas representa el escollo final en el proceso de regeneración (Fenner and Thompson 2005), y porque hay evidencia que los efectos maternos en la semilla pueden tener una influencia mayor que los del ambiente inmediato al momento de determinar los fenotipos de los jóvenes (Roach and Wulff 1987). Los mecanismos por los que pueden darse estos efectos maternos transgeneracionales son a través del aprovisionamiento de semillas, ARNm, proteínas, hormonas, metilación del ADN y modificación de histonas, además de efectos conjuntos entre éstos (Herman and Sultan 2011).

Bajo las condiciones menos estresantes para los árboles de Polylepis en el PNT (escenarios PRP y PRE) no se encontró ninguna relación entre la cobertura de dosel de las plantas madre y el crecimiento inicial de su progenie (Figura 6a). Sin embargo, en el escenario en que las especies exóticas estaban mezcladas con las nativas (escenario PME) sí hubo un efecto de la cobertura de dosel de la planta madre sobre el crecimiento inicial de su progenie. Además, en esta condición se observaron los valores más bajos de crecimiento inicial de Polylepis en el PNT. Estos resultados serían consecuencia de la competencia entre los árboles exóticos y nativos, posiblemente asociada con la producción de fotosintatos en condiciones estresantes. A mayor cobertura de dosel de la planta madre existe una mayor producción de fotosintatos. Estos fotosintatos se pueden usar para aumentar la cantidad de sustancias de reserva en las semillas, lo que, a su vez, afecta el crecimiento inicial de las plántulas (Roach and Wulff 1987). Por esta razón, los árboles exóticos en su condición más estresante (escenario PME) generaron un efecto materno de la cobertura de dosel de la planta madre sobre el crecimiento de su progenie.

Por otro lado, la sanidad de la planta madre modificó la relación entre el porcentaje de suelo desnudo y el crecimiento de su progenie 
(Figura 6b). En primer lugar, el crecimiento inicial de las plantas con sanidad baja e intermedia disminuyó ligeramente a medida que aumentó el porcentaje de suelo desnudo. Esto se puede explicar porque en suelos desnudos habría menor cantidad de nutrientes disponibles (mediados por la disponibilidad de agua $y$, posiblemente, por micorrizas; ver subtítulo Producción de semillas), que modificarían la manera en la que las plantas ubican sus nutrientes en su descendencia y, por ende, su desempeño en el crecimiento inicial (Vivas et al. 2015). Sin embargo, las plantas madre con buena sanidad presentaron hijos que respondieron de forma positiva a la disminución del porcentaje de suelo desnudo de las madres. Este resultado es inesperado porque el crecimiento inicial aumenta a medida que crece el porcentaje de suelo desnudo y, por lo tanto, disminuyen los nutrientes disponibles para la madre. Una segunda hipótesis es que en sitios con altos porcentaje de suelo desnudo también disminuiría la competencia con las plantas herbáceas y arbustivas (ver subtítulo Producción de semillas) y, por lo tanto, aumentarían los recursos para que las plantas madre los inviertan en las reservas de sus semillas. Se sabe que los progenitores pueden alterar características de sus hijos para responder a estreses particulares, y que dichas alteraciones podrían mejorar el crecimiento y éxito de sus hijos (Herman and Sultan 2011 y citas). Así que, bajo la condición de altos porcentajes de suelo desnudo, las plantas madre de Polylepis con alta sanidad producen semillas con mejores reservas y sus plántulas presentan mayor crecimiento inicial, hecho que les daría una ventaja adaptativa en relación a otras plántulas en campo.

Los efectos maternos reportados para el crecimiento de Polylepis en este estudio están de acuerdo con la literatura, que reporta que la altura de las plántulas, entre otras variables de crecimiento, tiene posibles efectos maternos citoplasmáticos (Roach and Wulff 1987 y citas), mediados por el aprovisionamiento de semillas, ARNm, hormonas y metabolitos secundarios, así como metilación de ADN y modificación de histonas (Herman and Sultan 2011). Los últimos autores indican que el peso de las semillas es un proxy confiable para el aprovisionamiento de las semillas. Si bien en el presente estudio no se pesaron las semillas (o frutos) de Polylepis, un estudio anterior con $P$. subtusalbida reportó que el peso de sus frutos secos fue 2.5 veces mayor en árboles pertenecientes a fragmentos rodeados de pajonal (escenario PRP) que en árboles presentes en bosques mixtos (escenario PME) (Gareca et al. 2007b), lo que sugiere que el aprovisionamiento de las semillas podría ser el posible mecanismo.

También se reportó un efecto materno sobre el crecimiento de individuos de $P$. australis en los que se observó mayor crecimiento en campo (hasta 3 años y medio) a mayor cobertura de Polylepis en un área con radio de 100 m del árbol madre (Renison and Cingolani 2002). Asimismo, se observó un crecimiento reducido en individuos de $P$. australis ubicados en fragmentos degradados, explicado posiblemente por la escasez de agua o falta de nutrientes en las plantas madre, que disminuyen las reservas y la calidad de las semillas y que podrían afectar al crecimiento de las plántulas (Renison et al. 2005). En este último caso, también se sugirió que el mecanismo de efecto materno de Polylepis sería el aprovisionamiento de semillas.

\section{Conclusiones}

Bajo los supuestos de polinización adecuada y compatibilidad genética, la producción de semillas de P. subtusalbida en el PNT, medida por el porcentaje de frutos con semillas completas por árbol, está limitada por la disponibilidad de nutrientes o de micorrizas, y no por las características propias de los árboles madre ni por los árboles exóticos. Además, en el jardín común, con condiciones homogéneas para la evaluación de la progenie, existe evidencia de un efecto de factores abióticos y bióticos presentes en el ambiente materno que se transmite y se expresa en el crecimiento inicial de la progenie, pero no sobre su germinación. Las especies exóticas generan un efecto de la cobertura de dosel de las plantas madre que se expresa en el crecimiento inicial de su descendencia, incluso en ausencia de las mismas. Por lo tanto, a) los árboles exóticos generaron un efecto del ambiente materno en la progenie del árbol nativo Polylepis subtusalbida y b) las condiciones del ambiente de la planta madre afectaron a su porcentaje de frutos con semillas completas por árbol y también el crecimiento inicial de su progenie en jardín común.

Se sugiere hacer experimentos de campo de traslocación de individuos en diferentes años, evaluar el supuesto de polinización adecuada y compatibilidad genética entre individuos, y experimentos de adición de nutrientes y remoción de especies herbáceas o arbustivas. También se podría estudiar el efecto de los 
depredadores y de la densidad de las especies exóticas sobre Polylepis. Para maximizar la cantidad de semillas y la velocidad de crecimiento de los individuos en un jardín común se recomienda seleccionar árboles sanos, evitar usar semillas provenientes de árboles presentes en sitios con abundante suelo desnudo y de aquellos ubicados en escenarios mixtos con especies exóticas. Sin embargo, con fines de conservación de diversidad genética es importante notar que los árboles descritos anteriormente posiblemente serán diferentes en términos genéticos a los demás y, por lo tanto, también será importante conservar su material genético. Asimismo, se apoya la recomendación de remover, ralear o podar los árboles exóticos que están dentro de los bosques nativos de Polylepis del PNT, como estrategia de conservación (Gareca et al. 2007c).

Agradecimientos. A los pobladores de Pajcha, Pintumayu y San Miguel por permitirnos hacer uso de los bosques estudiados. A la administración del Parque Nacional Tunari por permitirnos trabajar en el mismo. A BASFOR "Centro de Semillas Forestales" de la Escuela de Ciencias Forestales - Universidad Mayor de San Simón (UMSS) por facilitarnos las platabandas de germinación y crecimiento de plantines. O. Honnay y E. Matthysen apoyaron durante la preparación y ejecución del proyecto. Este trabajo fue parte del Proyecto "Permeabilidad de la matriz para el flujo genético entre bosques altoandinos amenazados de Polylepis" financiado por la Cooperación Belga VLIR-IUC-ZEIN2011RIP16 y con la autorización de investigación por parte de la Dirección General de Biodiversidad y Áreas Protegidas de Bolivia (cite MMAyAVMA-DGBAP No 619/12). Finalmente, agradecer a $S$. Arrázola y dos revisores anónimos quienes brindaron comentarios útiles y constructivos para mejorar la calidad y claridad del trabajo.

\section{REFERENCIAS}

Aguirre, L. F., R. Aguayo, O. Ruiz, and F. Navarro (eds.). 2007. Guía de los mamíferos, anfibios y reptiles del Parque Nacional Tunari. Centro de Biodiversidad y Genética. UMSS, Cochabamba, Bolivia.

Agreda, D. 2007. Estructura y composición florística de cinco tipos de bosques en la cuenca Pintumayu (Parque Nacional Tunari). Tesis de grado presentada para optar al Diploma Académico de Licenciatura en Biología. Carrera de Licenciatura en Biología. Universidad Mayor de San Simón, Cochabamba. Bolivia. Pp. 106.

Agreda, D. 2012. Caracterización de fragmentos de Polylepis y su matriz en el Parque Nacional Tunari. Informe final KEWIÑA-VLIR_001/2012. Universidad Mayor de San Simón, Centro de Biodiversidad y Genética, Cochabamba, Bolivia.

Anónimo. 2001. Parque Nacional Tunari: Área protegida, riquezas y límites. Boletín del Comité de Vigilancia del Parque Nacional Tunari. Cochabamba, Bolivia.

Domic, A. I., P. Bernhardt, R. Edens-Meier, G. R. Camilo, and J. M. Capriles. 2017. Pollination ecology of Polylepis tomentella (Rosaceae), an Andean anemophilous tree presenting a potential floral fungal infection. International Journal of Plant Sciences 178:512-521.

Everett, R. A. 2000. Patterns and pathways of biological invasions. Trends in Ecology and Evolution 15:177-178.

Fenner, M., and K. Thompson. 2005. The ecology of seeds. Cambridge University Press. Cambridge, United Kingdom.

Fjeldså. J., and M. Kessler. 1996. Conserving the biological diversity of Polylepis woodlands in the highland of Peru and Bolivia: A contribution to sustainable natural resource management in the Andes. Centre for research on the cultural and biological diversity of Andean rainforests (DIVA). DIVA technical report 11. NORDECO, Copenhaguen, Denmark.

Gareca, E. E., F. Vandelook, M. Fernández, M. Hermy, and O. Honnay. 2012. Seed germination, hydrothermal time models and the effects of global warming on a threatened high Andean tree species. Seed Science Research 22:287298.

Gareca, E. E., M. Hermy, J. Fjeldså, and O. Honnay. 2010. Polylepis woodland remnants as biodiversity islands in the Bolivian high Andes. Biodiversity and Conservation 19:3327-3346.

Gareca, E., Y. Martínez, C. Salazar, G. Arriarán, and L. F. Aguirre. 2007a. Efecto de Pinus radiata y Eucalyptus globulus sobre tres fases del ciclo de vida de Polylepis subtusalbida. Pp. 1401-1405 in J. Feyen, L. F. Aguirre and M. Moraes (eds.). Desarrollo, Medio Ambiente y Recursos Naturales: Sostenibilidad a Múltiples Niveles y Escalas. VLIR-UOS/UMSS, Cochabamba, Bolivia.

Gareca, E., Y. Martínez, F. Navarro, and J. Cahill. 2007b. Bases biológicas para un programa de reforestación con Polylepis subtusalbida en Cochabamba. Pp. 1413-1419 in J. Feyen, L. F. Aguirre and M. Moraes (eds.). Desarrollo, Medio Ambiente y Recursos Naturales: Sostenibilidad a Múltiples Niveles y Escalas. VLIR-UOS/UMSS, Cochabamba, Bolivia.

Gareca, E. E., Y. Y. Martínez, R. O. Bustamante, L. F. Aguirre, and M. M. Siles. 2007c. Regeneration patterns of Polylepis subtusalbida growing with the exotic trees Pinus radiata and Eucalyptus globulus at Parque Nacional Tunari, Bolivia. Plant Ecology 193:253-263.

Hensen, I. 1994. Estudios ecológicos y fenológicos sobre Polylepis besseri Hieron en la Cordillera Oriental Boliviana. Ecología en Bolivia 23:21-32. 
Herman, J. J., and S. E. Sultan. 2011. Adaptive transgenerational plasticity in plants: case studies, mechanisms, and implications for natural populations. Frontiers in Plant Science 2, 102:1-10.

Holl, K. D. 2012 Restoration of tropical forests. Pages 103-114 in J. van Andel and J. Aronson (eds.) Restoration ecology. Second Edition, Wiley-Blackwell, Oxford, United Kingdom.

Kessler, M. 1995. Present and potential distribution of Polylepis (Rosaceae) forests in Bolivia. Pp. 281-294 in S. P. Churchill, H. Balslev, E. Forero and J. L. Luteyn (eds.). Biodiversity and conservation of Neotropical montane forests: Proceedings of the Neotropical montane forest biodiversity and conservation symposium. New York Botanical Garden, New York, USA.

Kessler, M., and A. Schmidt-Lebuhn. 2006a. Taxonomical and distributional notes on Polylepis (Rosaceae). Organisms Diversity and Evolution 6:67-69.

Kessler, M., and A. N. Schmidt-Lebuhn. 2006b. Taxonomical and distributional notes on Polylepis (Rosaceae). Organisms Diversity and Evolution 6(Electronic Supplement 1):1-10.

Körner, C. 2003. Alpine plant life. Springer, Berlin, Germany.

Lavelle, P., and A. V. Spain. 2001. Soil ecology. Kluwer Academic Press. New York, USA.

Martínez, Y. Y., E. E. Gareca, L. Meneses, F. R. Castro, and L. F. Aguirre. 2006. Cambios en la morfología de frutos y germinación de semillas de Polylepis besseri por interacciones con especies exóticas en el Parque Nacional Tunari. Pp. 55 in P. Ojeda, M. Guardamiño, C. Aucca, J. Cahill and H. Arnal (eds.). II Congreso Internacional de Ecología y Conservación de bosques de Polylepis - Libro de resúmenes, Cusco, Perú.

Menoyo, E., D. Renison, and A. G. Becerra. 2009. Arbuscular mycorrhizas and performance of Polylepis australis trees in relation to livestock density. Forest Ecology and Management 258:2676-2682.

Mousseau, T. A., and C. W. Fox. 1998. The adaptive significance of maternal effects. Trends in Ecology and Evolution 13:403-407.

Naciones Unidas. 2015. Transformar nuestro mundo: la Agenda 2030 para el desarrollo sostenible. A/RES/70/1.

Navarro, G. 2015. Ecosistemas altimontanos de la Cordillera del Tunari. Pp. 178-194 in G. Navarro, L. F. Aguirre and M. M. Maldonado (eds.). Biodiversidad, ecología y conservación del Valle Central de Cochabamba. Centro de Biodiversidad y Genética, Universidad Mayor de San Simón. Cochabamba, Bolivia.

Navarro, G., J. A. Molina, and N. De la Barra. 2005. Classification of the high-Andean Polylepis forests in Bolivia. Plant Ecology 176:113-130.

Navarro, G., S. Arrázola, J. A. Balderrama, W. Ferreira, N. De la Barra, C. Antezana, I. Gómez, and M. Mercado. 2010. Diagnóstico del estado de conservación y caracterización de los bosques de Polylepis en Bolivia y su avifauna. Revista Boliviana de Ecología y Conservación Ambiental 28:1-35.

Paz, W. 1992. La necesidad de establecer la jurisdicción ambiental para garantizar las áreas protegidas y parques nacionales. Un modelo de estudio el Parque Nacional Tunari del departamento de Cochabamba. Tesis para optar el título de Lic. en Ciencias jurídicas y políticas. Universidad Mayor de San Simón, Cochabamba, Bolivia.

Pimentel, D., L. Lach, R. Zuniga, and D. Morrison. 2000. Environmental and economic costs associated with nonindigenous species in the United States. Bioscience 50:53-65.

Quinn, G., and M. Keough. 2002. Experimental design and data analysis for biologists. Cambridge University Press. Cambridge, United Kingdom.

R Core Team. 2017. R: A language and environment for statistical computing. R Foundation for Statistical Computing, Vienna, Austria. URL: www.R-project.org.

Reigosa, M., N. Pedrol, and A. Sanchéz. 2004. La Ecofisiología Vegetal. Una ciencia de síntesis. Editorial Thomson, Madrid, España.

Renison, D., and A. M. Cingolani. 2002. Evaluación de la supervivencia y crecimiento de plantines de Polylepis australis (Rosaceae) para la elección de plantas semilleras. Agriscientia 19:63-66.

Renison, D., I. Hensen, and A. M. Cingolani. 2004. Anthropogenic soil degradation affects seed viability in Polylepis australis mountain forests of central Argentina. Forest Ecology and Management 196:327-333.

Renison, D., A. M. Cingolani, R. Suárez, E. Menoyo, C. Coutsiers, A. Sobral, and I. Hensen. 2005. The restoration of degraded mountain woodlands: effects of seed provenance and microsite characteristics on Polylepis australis seedling survival and growth in Central Argentina. Restoration Ecology 13:129-137.

Rico, A. 1996. Efectos indirectos derivados de actividades del programa de repoblamiento forestal en el Parque Nacional Tunari. Cordeco-Cotesu, Cochabamba, Bolivia.

Roach, D. A., and R. D. Wulff. 1987. Maternal effects in plants. Annual Review in Ecology and Systematics 18:209235.

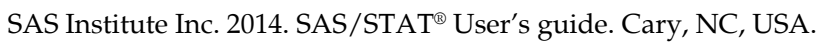

Seltman, P., I. Leyer, D. Renison, and I. Hensen. 2007. Variation of seed mass and its effects on germination in Polylepis australis: implications for seed collection. New Forests 33:171-181.

SERNAP. 2000. Información técnica del Sistema Nacional de Áreas Protegidas de Bolivia. Editorial Plural. La Paz, Bolivia.

Simpson, B. B. 1979. A Revision of the Genus Polylepis (Rosaceae: Sanguisobeae). Smithsonian Contributions to Botany 43. Washington, USA.

Thomas, E., R. Jalonen, J. Loo, D. Boshier, L. Gallo, S. Cavers, S. Bordács, P. Smith, and M. Bozzano. 2014. Genetic considerations in ecosystem restoration using native tree species. Forest Ecology and Management 333:66-75. 
Vega, C., J. C. Bermejo, P. R. Koya, G. Villegas, J. Quezada, and C. López. 2016. La Keñua (Polylepis) - Árbol altoandino como alternativa ornamental para reforestación en áreas verdes urbanas del municipio de La Paz. Unidad de Biotecnología Vegetal, Universidad Mayor de San Andrés, La Paz, Bolivia.

Vivas, M., M. Kemler, and B. Slippers. 2015. Maternal effects on tree phenotypes: considering the microbiome. Trends in Plant Science 20:541-544.

Weiher, E., A. Werf, K. Thompson, M, Roderick, E. Garnier and O. Eriksson. 1999. Challenging Theophrastus: a common core list of plant traits for functional ecology, Journal of Vegetation Science 10:609-620

White, T. L., W. T. Adams, and D. B. Neale. 2007. Forest genetics. CAB International, London, United Kingdom.

Wickham, H. 2009. ggplot2: elegant graphics for data analysis. Springer, New York, USA.

Wolf, B. J., and M. J. Wade. 2009. What are maternal effects (and what they are not). Philosophical Transactions of the Royal Society B 364:1107-1115.

Zahawi, R. A., and K. D. Holl. 2014. Evaluation of different tree propagation methods in ecological restoration in the Neotropics. Pp. 85-96 in M. Bozzano, R. Jalonen, E. Thomas, D. Boshier, L. Gallo, S. Cavers, S. Bordács, P. Smith and J. Loo (eds.). Genetic considerations in ecosystem restoration using native tree species. FAO, Roma, Italy. 